\title{
Spin-orbit coupling and spin relaxation in phosphorene: Intrinsic versus extrinsic effects
}

\author{
Marcin Kurpas, Martin Gmitra, and Jaroslav Fabian \\ Institute for Theoretical Physics, University of Regensburg, Regensburg 93040, Germany
}

(Received 14 May 2016; published 13 October 2016)

\begin{abstract}
First-principles calculations of the essential spin-orbit and spin relaxation properties of phosphorene are performed. Intrinsic spin-orbit coupling induces spin mixing with the probability of $b^{2} \approx 10^{-4}$, exhibiting a large anisotropy, following the anisotropic crystalline structure of phosphorene. For realistic values of the momentum relaxation times, the intrinsic (Elliott-Yafet) spin relaxation times are hundreds of picoseconds to nanoseconds. Applying a transverse electric field (simulating gating and substrates) generates extrinsic $C_{2 v}$ symmetric spin-orbit fields in phosphorene, which activate the D'yakonov-Perel' mechanism for spin relaxation. It is shown that this extrinsic spin relaxation also has a strong anisotropy and can dominate over the Elliott-Yafet one for strong enough electric fields. Phosphorene on substrates can thus exhibit an interesting interplay of both spin-relaxation mechanisms, whose individual roles could be deciphered using our results.
\end{abstract}

DOI: 10.1103/PhysRevB.94.155423

Phosphorene is a monolayer of black phosphorus [1-5] exhibiting a direct band gap of $2 \mathrm{eV}$ [6,7] and large anisotropic mobility [6,8,9]. Unlike graphene, phosphorene is a semiconductor, and unlike two-dimensional transition-metal dichalcogenides, which are semiconductors too, phosphorene is distinctly anisotropic thanks to its puckered atomic structure. The semiconductor property makes phosphorene suitable for electronic [10] and spintronics applications [11,12], in particular, for bipolar spin diodes and transistors [13], while the anisotropy enables directional control of the essential spin properties, such as spin-orbit coupling and spin relaxation. In contrast to graphene, whose spin properties are by now well established [14], there is no unified picture of the spin-orbit coupling and spin relaxation in phosphorene.

Phosphorene can be extracted from black phosphorus by mechanical $[8,10,15]$ or liquid $[16,17]$ cleavage techniques. Inside phosphorene layers, each phosphorus atom is covalently bonded with three adjacent phosphorus atoms to form a puckered honeycomb structure due to $s p^{3}$ hybridization (see Fig. 1). The puckered structure can be viewed as a two-layer system in which the bonding energy is dominated by the in-plane bonds ( $p p \sigma$ and $p p \pi)$ that are much stronger than the bonds connecting the two sublayers $(p p \pi)$ [18]. Similarly to graphene, the edges of phosphorene form zigzag (along the $x$ axis) and armchair (along the $y$ axis) chains [see Fig. 1(b)].

Black phosphorus is described by the nonsymmorphic $D_{2 h}$ point group being isomorphic with the $C_{m c a}$ space group. Phosphorene shares the same point-group symmetry as its bulk counterpart. Both structures have inversion symmetry leading to spin-degenerate eigenstates. Spin-orbit coupling leads to the spin mixing of the Pauli spinors-the intrinsic effect. When inversion symmetry of phosphorene is broken by an applied transverse electric field $\mathbf{E}$ or a substrate, the point group is reduced to nonsymmorphic $C_{2 v}$, with the principal $C_{2}$ axis parallel to the direction of the electric field and two mirror planes $\sigma_{x z}$ and $\sigma_{y z}$ [Figs. 1(a) and 1(b)]. In this case the spin degeneracy is lifted-the extrinsic (Rashba) effect.

From the spintronics perspective two questions are particularly important to address: (i) what is the intrinsic and extrinsic spin-orbit coupling (SOC) in phosphorene and (ii) what are the relevant spin-relaxation time scales. The first question has been partially answered by Popović et al. [19], who showed that the extrinsic Rashba effect, due to external electric fields, is anisotropic with respect to the two principal directions in the crystal. The second question has been addressed within $k \cdot p$ theory [18] for the intrinsic effects only.

Here we employ first-principles calculations to address both questions, providing state-of-the-art, most realistic results for the extrinsic and intrinsic effects. First, we find that intrinsic SOC lifts degeneracy of the valence and conduction bands at the $S$ point, by splitting the bands at about $17.5 \mathrm{meV}$ and $14 \mathrm{meV}$, respectively. The extrinsic Rashba SOC is much weaker, of the order of tens of $\mu \mathrm{eV}$ close to the $\Gamma$ point (for electric fields of $1 \mathrm{~V} / \mathrm{nm}$ ), and is found to be significantly anisotropic for the valence band only. Second, we predict the spin lifetime in phosphorene to be hundreds of picoseconds up to nanoseconds, for the experimentally relevant mobilities. We find that for no and small electric fields up to $\mathrm{E} \approx 2.5 \mathrm{~V} / \mathrm{nm}$ and carrier densities up to $8 \times 10^{12} \mathrm{~cm}^{-2}$, the dominant spinrelaxation mechanism is the Elliott-Yafet [20,21]. For the in-plane spin orientation the relaxation is almost twice slower than for spins oriented out of plane. By increased transverse electric field the D'yakonov-Perel' [22] mechanism starts to be the most effective. For carrier density $n \approx 3 \times 10^{12} \mathrm{~cm}^{-2}$, it overtakes the Elliott-Yafet at $E=4 \mathrm{~V} / \mathrm{nm}$ for holes and $E=5 \mathrm{~V} / \mathrm{nm}$ for electrons. As a result, the substrates can be essential for spin dynamics in phosphorene due to an interplay between the D'yakonov-Perel' and the Elliott-Yafet relaxation mechanisms.

Intrinsic phosphorene belongs to the family of centrosymmetric crystals for which the dominant spin-relaxation mechanism is the Elliott-Yafet scattering [20,21]. The strength of the scattering can be quantified by the spin admixture coefficient $b^{2}$ [20], which is a signature of intrinsic spin-orbit coupling. The time-reversal and spaceinversion symmetries require two Bloch states $\Psi_{n, k}^{\uparrow}(\mathbf{r})$ and $\Psi_{n, k}^{\downarrow}(\mathbf{r})$ of the same band $n$ and momentum $k$ to be degenerate (Kramer's doublets). Due to spin-orbit coupling, these states are mixtures of spin-up $|\uparrow\rangle$ and spin-down $|\downarrow\rangle$ Pauli spinors: $\Psi_{n, \mathbf{k}}^{\uparrow}(\mathbf{r})=\left[a_{n, \mathbf{k}}(\mathbf{r})|\uparrow\rangle+b_{n, \mathbf{k}}(\mathbf{r})|\downarrow\rangle\right] e^{i \mathbf{k r}}$, $\Psi_{n, \mathbf{k}}^{\downarrow}(\mathbf{r})=\left[a_{n,-\mathbf{k}}^{*}(\mathbf{r})|\downarrow\rangle-b_{n,-\mathbf{k}}^{*}(\mathbf{r})|\uparrow\rangle\right] e^{i \mathbf{k r}}$. For a generic Bloch state the modulation functions $a_{n, \mathbf{k}}(\mathbf{r})$ and $b_{n, \mathbf{k}}(\mathbf{r})$ are selected to diagonalize the spin magnetic moment along the chosen 
(a)

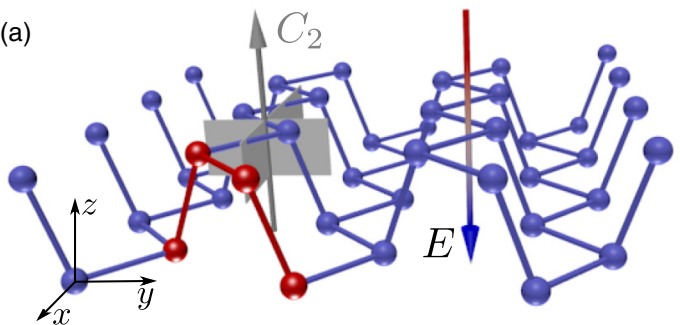

(b)

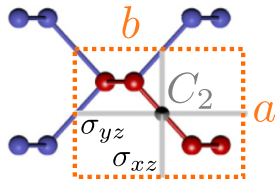

(c)

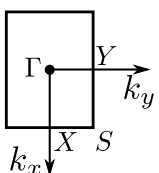

FIG. 1. Essence of phosphorene crystal structure. (a) Schematic of a single layer of black phosphorus with drawn symmetry axis $C_{2}$, mirror planes $\sigma_{x z}$ and $\sigma_{y z}$, and the vector of electric field $E$. The red-colored atoms form the unit cell of phosphorene, and the gradient of color of the electric field vector corresponds to higher (red) and lower (blue) electrostatic potential. (b) Top view to the structure. The unit cell is marked by orange dashed line. (c) The first Brillouin zone of phosphorene with labels of high symmetry points.

direction, corresponding to the injected spin in experiment; for weak spin-orbit coupling, $b_{n, \mathbf{k}}(\mathbf{r})$ stands for the small spin component being admixed to the large spin component $a_{n, \mathbf{k}}(\mathbf{r})$, i.e., $\left|a_{n, \mathbf{k}}(\mathbf{r})\right|^{2} \gg\left|b_{n, \mathbf{k}}(\mathbf{r})\right|^{2}$. The Elliott-Yafet scattering parameter $b^{2}$ is defined as the Fermi surface average of the unit-cell integrated admixture coefficient $b_{n, \mathbf{k}}^{2}$,

$$
\begin{gathered}
b^{2}=\left\langle b_{n, \mathbf{k}}^{2}\right\rangle=\left[\rho\left(\varepsilon_{\mathrm{F}}\right) S_{\mathrm{BZ}}\right]^{-1} \int_{\mathrm{FS}} b_{n, \mathbf{k}}^{2} /\left|\hbar v_{\mathrm{F}}\left(\varepsilon_{\mathrm{F}}\right)\right| d k, \\
b_{n, \mathbf{k}}^{2}=\int\left|b_{n, \mathbf{k}}(\mathbf{r})\right|^{2} d^{3} r,
\end{gathered}
$$

where $0 \leqslant b^{2} \leqslant 0.5, \rho\left(\varepsilon_{\mathrm{F}}\right)$ is the density of states per spin at the Fermi level, $v_{\mathrm{F}}$ is the Fermi velocity, and $S_{\mathrm{BZ}}$ is the area of the Brillouin zone. If the scattering potential is spin independent (scalar impurities and phonons), the intrinsic SOC leads to spin-flip scattering. The Elliott-Yafet mechanism gives for the spin-relaxation rate $[20,23]$

$$
\tau_{\mathrm{s}, \mathrm{EY}}^{-1} \approx 4 b^{2} \tau_{p}^{-1}
$$

where $\tau_{p}^{-1}$ is the momentum relaxation rate.

Extrinsic effects appear once the space-inversion symmetry gets broken, e.g., by a substrate or external fields. The spin degeneracy gets lifted and another spin-relaxation mechanism appears: D'yakonov-Perel' [22]. This mechanism can be viewed as a motional narrowing of the spin precession in a fluctuating (due to momentum scattering) emerging spin-orbit field $\Omega_{\mathbf{k}}$, which is related to the spin splitting as

$$
H_{\mathrm{ex}}=\frac{\hbar}{2} \Omega_{\mathbf{k}} \cdot \boldsymbol{\sigma},
$$

where $\sigma$ is the vector of Pauli matrices. In the relevant limit of small correlation times $\left(\Omega \tau_{p} \ll 1\right)$, i.e., when the precession angle between the scattering events is small, the spin-relaxation rate becomes [22]

$$
\tau_{\mathrm{s}, \mathrm{DP}}^{-1}=\Omega_{\perp}^{2} \tau_{p},
$$

where $\Omega_{\perp}^{2}=\left\langle\Omega_{\mathbf{k}, \perp}^{2}\right\rangle$ is the Fermi contour average of the squared spin-orbit field projected to the plane perpendicular to the spin orientation.

In our first-principles calculations we used the initial crystal structure parameters from Ref. [1] for bulk black phosphorus. A sheet of phosphorene was placed in vacuum of $20 \AA$ and fully relaxed using a quasi-Newton variable-cell scheme as implemented in the QUANTUM ESPRESSO [24] package. Positions of atoms have been relaxed in all directions, with the force convergence threshold $10^{-4}$ Ry/a.u. (atomic unit) and total energy convergence condition $10^{-5}$ Ry/a.u. The norm-conserving pseudopotential, with kinetic energy cutoffs of $70 \mathrm{Ry}$ and $280 \mathrm{Ry}$ for the wave function and charge density, respectively, has been used along with the Perdew-BurkeErnzerhof exchange-correlation functional [25]. Obtained structural parameters are summarized in Ref. [26].

Further electronic structure calculations have been performed using the full-potential linearized augmented planewave method as implemented in the all-electron code package WIEN2K [27]. Self-consistency has been achieved for a $16 \times 12 \times 1$ Monkhorst-Pack $k$-point grid with $151 k$-points in the irreducible wedge of the Brillouin zone. SOC has been included fully relativistically for core electrons, while five valence electrons have been treated within the second variational step method [28]. For the calculations with the transverse electric field, we considered a vacuum size of $25 \AA$. It is known that standard DFT methods underestimate the band gaps of semiconductors. Theoretical band gaps of phosphorene spread between 0.7 and $2.2 \mathrm{eV}$, depending on the method of calculations [8,9,29-32]. On the other hand, recent experimental reports suggest the band gap of phosphorene to be about $2 \mathrm{eV}[7,33]$. It has been reported for standard semiconductors [34] that the underestimation of the band gap significantly impairs SOC effects. Therefore, to consider a realistic band gap we perform the calculations with an undressed local-density approximation (LDA) functional along with the modified Becke-Johnson (mBJ) potential [35] parametrized to give the band gap of $2.17 \mathrm{eV}$.

The calculated band structure of phosphorene is shown in Fig. 2. We get a direct gap at the zone center. Some density functional theory (DFT) calculations [19,29,36] report a nearly indirect band gap, with a somewhat displaced valence-band maximum. The valence band in the vicinity of the $\Gamma$ point along $k_{x}$ is nearly dispersionless, while it is very dispersive in the $k_{y}$ direction. A similar, but substantially smaller, dispersion anisotropy is seen in the conduction band. Close to the $\Gamma$ point both, the valence and the conduction band have mainly $p_{z}$ orbital character, the latter having a small admixture of $p_{y}$ orbitals [26]. The next conduction band minimum appears at energy $50 \mathrm{meV}$ above the global conduction band minimum (in direction towards the $X$ point) and consists mainly of $p_{x}$ and $p_{y}$ orbitals with an admixture of $d_{x^{2}-y^{2}}$ electrons. The small distance of this band to the conduction band minimum is reflected as an increase of the slope in the carrier density $n\left(\varepsilon_{\mathrm{F}}\right)$ shown in Fig. 2(e) as a function of the Fermi level $\varepsilon_{\mathrm{F}}$. For the valence band the carrier density is a smooth quadratic 


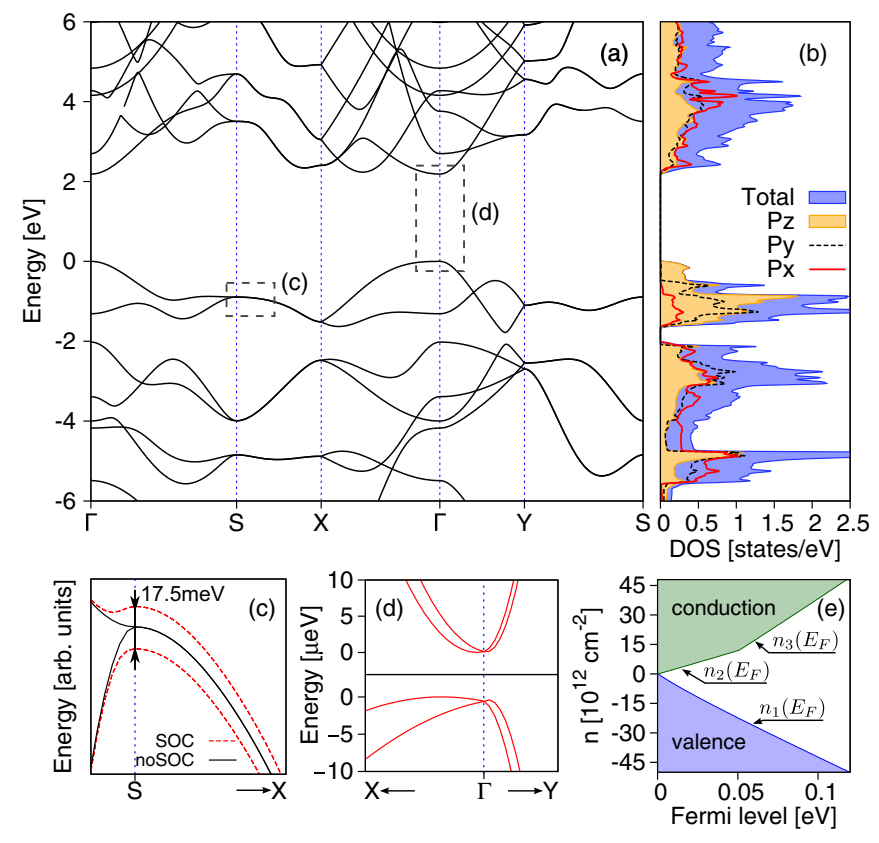

FIG. 2. Calculated electronic properties of phosphorene using the LDA $+\mathrm{mBJ}$ (local density approximation-modified Becke-Johnson) exchange-correlation functional. (a) Band structure along high symmetry lines. The marked areas (c) and (d) are zoomed in the bottom row of the figure. (b) p-orbital resolved (yellow-filled curve, solid and dashed lines) and total (blue-filled curve) density of states. (c) Splitting of the valence band along the $S-X$ line due to intrinsic spin-orbit coupling. (d) Sketch of the extrinsic (Rashba) spin-orbit coupling effect to the band structure close to $\Gamma$. (e) Carrier concentration as a function of the Fermi level. The value $n=0$ corresponds to the Fermi level at the valence (conduction) band maximum (minimum). Positive values of $n$ correspond to electron, negative to hole doping. Empirical fits to the first-principles results give $n_{1}\left(\varepsilon_{\mathrm{F}}\right)=-243.3 \varepsilon_{\mathrm{F}}^{2}-441.4 \varepsilon_{\mathrm{F}}+0.86$, $n_{2}\left(\varepsilon_{\mathrm{F}}\right)=238.9 \varepsilon_{\mathrm{F}}-0.14, n_{3}\left(\varepsilon_{\mathrm{F}}\right)=537.9 \varepsilon_{\mathrm{F}}-15.52$, where $\varepsilon_{\mathrm{F}}$ is the Fermi level in electronvolts and density $n$ in $10^{12} \mathrm{~cm}^{-2}$.

function of $\varepsilon_{\mathrm{F}}$. Empirical fits for the dependence $n\left(\varepsilon_{F}\right)$, which should be useful for interpreting experiments, are given in the caption to Fig. 2.

Intrinsic effects. We first discuss the intrinsic SOC and the Elliott-Yafet spin relaxation. The intrinsic SOC in phosphorene is relatively strong but does not modify substantially the band structure close to the band gap. The orbital degeneracy of the fourfold-degenerate bands [see inset to Fig. 2(c)] is split into two pairs of spin-degenerate bands. The splitting is maximal at the $S$ point, $17.5 \mathrm{meV}$ and $14 \mathrm{meV}$ for valence and conduction band, respectively, gradually decreasing towards the time-reversal points $X$ and $Y$. The states at the Brillouin zone boundaries stay degenerate due to nonsymmorphicity of the $D_{2 h}$ group [37].

The important effect of the intrinsic SOC is the spin mixing, quantified by $b_{\mathbf{k}}^{2}$. In Figs. 3(a) and 3(b) we show the distribution of spin-mixing parameter $b_{\mathbf{k}}^{2}$ in the first Brillouin zone (BZ) of phosphorene for the spin quantization axis oriented perpendicular to the two-dimensional plane. The other spin orientations are discussed in Ref. [26]. For momenta corresponding to anticrossings and at the BZ edges (except
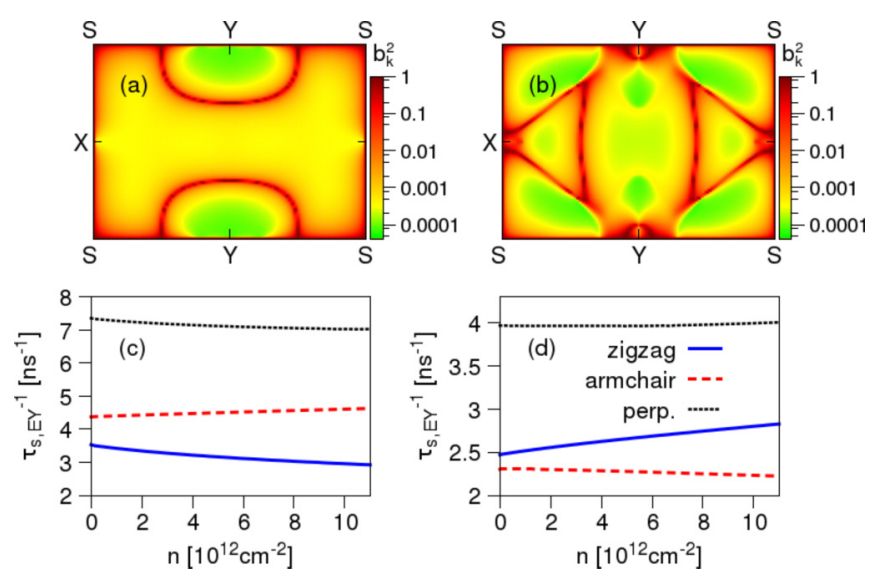

FIG. 3. Intrinsic spin-orbit coupling effects in phosphorene. (a) Momentum-resolved spin-mixing parameter $b_{\mathbf{k}}^{2}$ for the valence band and out-of-plane spin direction. (b) Same as in (a) but for the conduction band. (c) Elliott-Yafet spin-relaxation rates for valence electrons, for indicated spin directions, as a function of the carrier density. (d) Same as in (c) but for the conduction band. Constant typical momentum relaxation time $\tau_{p}=100 \mathrm{fs}$ is assumed.

the points $X$ and $Y$ for which $b_{\mathbf{k}}^{2}$ is zero) the values of $b_{\mathbf{k}}^{2}$ are close to $\frac{1}{2}$. The Bloch eigenstates here are fully spin mixed, forming spin hot spots $[23,38]$. At the zone center $b_{\mathbf{k}}^{2}$ is about $10^{-4}$. Perturbation theory gives that $b$ is roughly the ratio of the intrinsic spin-orbit coupling (order $10 \mathrm{meV}$ ) and the band gap (order $1 \mathrm{eV}$ ); thus $b \approx 0.01$ matches well to the calculated first-principles value of $b^{2} \approx 10^{-4}$. For comparison, a recent estimate based on $k \cdot p$ theory gives $b^{2}$ between $10^{-6}$ and $10^{-5}$ [18].

Knowing $b^{2}$ we now calculate the Elliott-Yafet spinrelaxation rates using Eq. (3). For the momentum relaxation we take the typical experimental value of $\tau_{p}=100 \mathrm{fs}$. The results can be easily rescaled for the actual experimental mobilities. Calculated $\tau_{\mathrm{s}, \mathrm{EY}}^{-1}$ as a function of carrier density $n\left(\varepsilon_{\mathrm{F}}\right)$, for valence and conduction bands and different spin quantization axes, are shown in Figs. 3(c) and 3(d). The relaxation rates are almost independent of $n$, which follows $b^{2}$ since we use a constant momentum relaxation time. The monotonicity of $\tau_{\mathrm{s}, \mathrm{EY}}^{-1}$ is then unambiguously determined by $b^{2}$. The spin-relaxation rates of holes are greater than those of electrons. Most striking is the strong anisotropy. The largest spin relaxation is for out-of-plane spins, which relax roughly twice as fast as the in-plane spins. We predict the longest spin lifetimes for armchair-oriented spins in the conduction band and zigzag-oriented spins in the valence band. In the recent $k \cdot p$ theory [18], the estimated ratio between the spin-relaxation rate for out-of-plane to in-plane spins was $\sim 4$, which is an overestimation in view of our first-principles results, but is in qualitative agreement. Similar anisotropies in Elliott-Yafet spin lifetimes were also predicted for anisotropic bulk materials and thin metallic films [39-41].

Extrinsic effects. In realistic situations phosphorene sits on a substrate or is studied in a gating electric field which breaks space-inversion symmetry $\left(D_{2 h} \longrightarrow C_{2 v}\right)$. An extrinsic Rashba spin-orbit field emerges, lifting the spin degeneracy, $\varepsilon_{\mathbf{k} \uparrow} \neq \varepsilon_{\mathbf{k} \downarrow}$, according to Eq. (4), except at time-reversal 

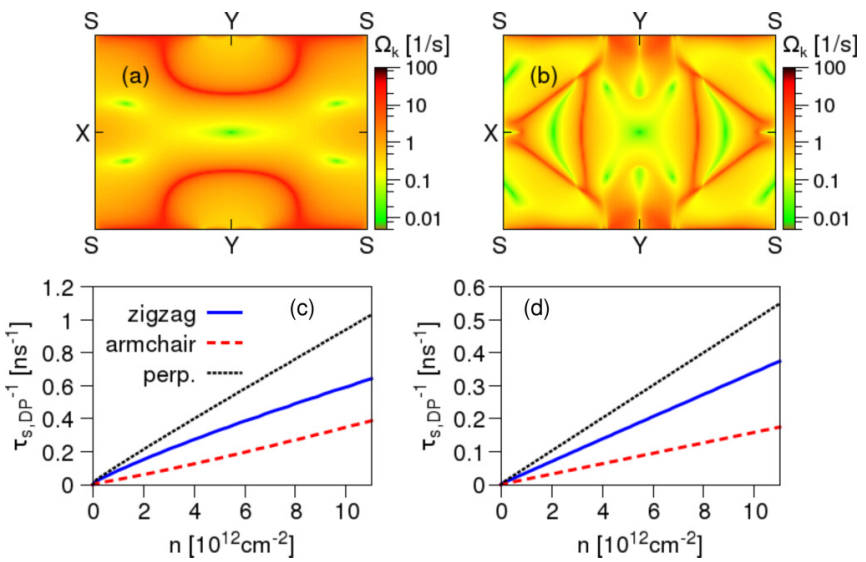

FIG. 4. Extrinsic spin-orbit coupling effects in phosphorene. (a) Spin-orbit field magnitude $\Omega_{\mathbf{k}}$ in the first Brillouin zone for the valence band and transverse electric field of $E=1 \mathrm{~V} / \mathrm{nm}$. (b) Same as in (a) but for the conduction band. Calculated D'yakonov-Perel' relaxation rates, assuming $\tau_{p}=100 \mathrm{fs}$, as a function of carrier density for (c) valence band and (d) conduction band for indicated spin directions. For spins perpendicular to the phosphorene plane empirical fitting gives $\tau_{\mathrm{s}, \mathrm{DP}}^{-1}(n)\left[\mathrm{ns}^{-1}\right] \approx 0.093 n$ for the valence band and $\tau_{\mathrm{s}, \mathrm{DP}}^{-1}(n)\left[\mathrm{ns}^{-1}\right] \approx 0.05 n$ for the conduction band, where $n$ is in units of $10^{12} \mathrm{~cm}^{-2}$.

invariant points. Emerged spin-orbit fields give rise to spin relaxation due to the D'yakonov-Perel' mechanism, which competes with the Elliott-Yafet spin-flip scattering. Here we model the symmetry breaking by applying a transverse electric field, all within the first-principles calculations, of $1 \mathrm{~V} / \mathrm{nm}$. In Figs. 4(a) and 4(b) we plot the spin-orbit field $\Omega_{\mathbf{k}}$ magnitude over the first Brillouin zone. Similarly to $b_{\mathbf{k}}^{2}$, the values of $\Omega_{\mathbf{k}}$ are peaked at the band anticrossings and at the BZ edges. At the time-reversal points $\Omega_{\mathbf{k}}$ is zero.

We note that for a bare Perdew-Burke-Ernzerhof exchangecorrelation functional [25] (band gap $E_{g} \approx 1 \mathrm{eV}$ ), the Rashba spin-orbit coupling due to external electric fields exhibits a strong anisotropy in the valence as well as in the conduction band [19]. Our calculations show that increasing the band gap to the experimental value $\approx 2 \mathrm{eV}$ removes the anisotropy from the conduction band, while it is preserved for the valence band.

To obtain the spin-relaxation rates for the D'yakonov-Perel' mechanism, we resolve the coordinate components of the vector spin-orbit fields $\Omega$ which lie in the phosphorene plane: $\Omega_{x}$ along $x$ (zigzag) and $\Omega_{y}$ along $y$ (armchair) directions. We extract these components by fitting an effective $C_{2 v}$ symmetric spin-orbit coupling Hamiltonian to the first-principles data [26]. In Figs. 4(c) and 4(d) we show the calculated spin-relaxation rates using Eq. (5), assuming $E=1 \mathrm{~V} / \mathrm{nm}$ and $\tau_{p}=100 \mathrm{fs}$. The spin lifetime is exceptionally long, of a few nanoseconds, and exceeds the lifetime from the Elliott-Yafet mechanism. The relaxation rates for conduction electrons are twice smaller than for the valence electrons. With a growing electric field, the D'yakonov-Perel' mechanism becomes more significant. For the valence band, it surpasses ElliottYafet's for $n \gtrsim 6 \times 10^{12} \mathrm{~cm}^{-2}, n \gtrsim 3 \times 10^{12} \mathrm{~cm}^{-2}$, and $n \gtrsim$ $2 \times 10^{12} \mathrm{~cm}^{-2}$ for electric fields $E=3 \mathrm{~V} / \mathrm{nm}, E=4 \mathrm{~V} / \mathrm{nm}$, and $E=5 \mathrm{~V} / \mathrm{nm}$, respectively [26]. For the conduction band the transitions happen for slightly higher carrier densities. For electric fields $E=5 \mathrm{~V} / \mathrm{nm}$ the D'yakonov-Perel' spinrelaxation rates are $\approx 23$ times greater than for $E=1 \mathrm{~V} / \mathrm{nm}$. Similarly to $b^{2}$, the spin-orbit field $\Omega^{2}$ reveals a strong anisotropy. As a result the in-plane spins relax about $1.5-3$ times slower than the out-of-plane ones.

In summary, we have studied intrinsic and extrinsic spinorbit coupling and spin-relaxation mechanisms in phosphorene. The Elliott-Yafet spin relaxation gives spin lifetimes less than nanoseconds for experimentally relevant samples. The D'yakonov-Perel' mechanism matters at large electric fields. The lifetimes exhibit a large anisotropy for in-plane and out-of-plane spin orientations.

We acknowledge funding from Deutsche Forshungsgemeinschaft via SPP 1538 and SFB 689, NCN DEC2013/11/B/ST3/00824 and the EU Seventh Framework Programme under Grant Agreement No. 604391 Graphene Flagship. The authors acknowledge the Gauss Centre for Supercomputing e.V. (www.gauss-centre.eu) for computing time on the GCS Supercomputer SuperMUC at Leibniz Supercomputing Centre (LRZ, www.lrz.de).
[1] A. Brown and S. Rundqvist, Acta Crystallogr. 19, 684 (1965).

[2] L. Cartz, S. R. Srinivasa, R. J. Riedner, J. D. Jorgensen, and T. G. Worlton, J. Chem. Phys. 71, 1718 (1979).

[3] R. W. Keyes, Phys. Rev. 92, 580 (1953).

[4] Y. Maruyama, S. Suzuki, K. Kobayashi, and S. Tanuma, Physica B+C 105, 99 (1981).

[5] S. Narita, Y. Akahama, Y. Tsukiyama, K. Muro, S. Mori, S. Endo, M. Taniguchi, M. Seki, S. Suga, A. Mikuni, and H. Kanzaki, Physica B+C 117, 422 (1983).

[6] A. Castellanos-Gomez, L. Vicarelli, E. Prada, J. O. Island, K. L. Narasimha-Acharya, S. I. Blanter, D. J. Groenendijk, M. Buscema, G. A. Steele, J. V. Alvarez, H. W. Zandbergen, J. J. Palacios, and H. S. J. v. d. Zant, 2D Mater. 1, 025001 (2014).

[7] L. Liang, J. Wang, W. Lin, B. G. Sumpter, V. Meunier, and M. Pan, Nano Lett. 14, 6400 (2014).
[8] H. Liu, A. T. Neal, Z. Zhu, Z. Luo, X. Xu, D. Tománek, and P. D. Ye, ACS Nano 8, 4033 (2014).

[9] J. Qiao, X. Kong, Z.-X. Hu, F. Yang, and W. Ji, Nat. Commun. 5, 4475 (2014).

[10] L. Li, Y. Yu, G. J. Ye, Q. Ge, X. Ou, H. Wu, D. Feng, X. H. Chen, and Y. Zhang, Nat. Nanotechnol. 9, 372 (2014).

[11] I. Žutić, J. Fabian, and S. Das Sarma, Rev. Mod. Phys. 76, 323 (2004).

[12] J. Fabian, A. Matos-Abiague, C. Ertler, P. Stano, and I. Žutić, Acta Phys. Slovaca 57, 565 (2007).

[13] I. Žutić, J. Fabian, and S. Erwin, IBM J. Res. Dev. 50, 121 (2006).

[14] W. Han, R. K. Kawakami, M. Gmitra, and J. Fabian, Nat. Nanotechnol. 9, 794 (2014). 
[15] F. Xia, H. Wang, and Y. Jia, Nat. Commun. 5, 4458 (2014).

[16] J. R. Brent, N. Savjani, E. A. Lewis, S. J. Haigh, D. J. Lewis, and P. O'Brien, Chem. Commun. 50, 13338 (2014).

[17] J. Kang, J. D. Wood, S. A. Wells, J.-H. Lee, X. Liu, K.-S. Chen, and M. C. Hersam, ACS Nano 9, 3596 (2015).

[18] P. Li and I. Appelbaum, Phys. Rev. B 90, 115439 (2014).

[19] Z. S. Popović, J. M. Kurdestany, and S. Satpathy, Phys. Rev. B 92, 035135 (2015).

[20] R. J. Elliott, Phys. Rev. 96, 266 (1954).

[21] Y. Yafet, in Solid State Physics, edited by F. Seitz and D. Turnbull (Academic Press, New York, 1963), Vol. 14.

[22] M. I. D’yakonov and V. I. Perel', Fiz. Tverd. Tela 13, 3581 (1971) [Sov. Phys. Solid State 13, 3023 (1972)].

[23] J. Fabian and S. Das Sarma, Phys. Rev. Lett. 81, 5624 (1998).

[24] P. Giannozzi, S. Baroni, N. Bonini, M. Calandra, R. Car, C. Cavazzoni, D. Ceresoli, G. L. Chiarotti, M. Cococcioni, I. Dabo, A. Dal Corso, S. de Gironcoli, S. Fabris, G. Fratesi, R. Gebauer, U. Gerstmann, C. Gougoussis, A. Kokalj, M. Lazzeri, L. MartinSamos et al., J. Phys.: Condens. Matter 21, 395502 (2009).

[25] J. P. Perdew, K. Burke, and M. Ernzerhof, Phys. Rev. Lett. 77, 3865 (1996).

[26] See Supplemental Material at http://link.aps.org/supplemental/ 10.1103/PhysRevB.94.155423 for structural parameters, calculation of effective SOC Hamiltonian parameters, and spin-orbit fields. The distribution of the spin-mixing parameter $b_{\mathbf{k}}^{2}$ in the first Brillouin zone, the Elliott-Yafet spin-scattering parameter $b^{2}$, comparison of relaxation mechanisms for several values of electric fields, and orbital decomposition of the band structure are also discussed.

[27] P. Blaha, K. Schwarz, G. K. H. Madsen, D. Kvasnicka, and J. Luitz, WIEN2K, An Augmented Plane Wave + Local
Orbitals Program for Calculating Crystal Properties (Karlheinz Schwarz, Techn. Universität Wien, Austria, 2001).

[28] D. J. Singh and L. Nordstrom, Planewaves, Pseudopotentials, and the LAPW Method (Springer, New York, 2006).

[29] A. S. Rodin, A. Carvalho, and A. H. Castro Neto, Phys. Rev. Lett. 112, 176801 (2014).

[30] T. Hu, Y. Han, and J. Dong, Nanotechnology 25, 455703 (2014).

[31] A. N. Rudenko and M. I. Katsnelson, Phys. Rev. B 89, 201408 (2014).

[32] V. Tran, R. Soklaski, Y. Liang, and L. Yang, Phys. Rev. B 89, 235319 (2014).

[33] X. Wang, A. M. Jones, K. L. Seyler, V. Tran, Y. Jia, H. Zhao, H. Wang, L. Yang, X. Xu, and F. Xia, Nat. Nanotechnol. 10, 517 (2015).

[34] A. N. Chantis, M. van Schilfgaarde, and T. Kotani, Phys. Rev. Lett. 96, 086405 (2006).

[35] F. Tran and P. Blaha, Phys. Rev. Lett. 102, 226401 (2009).

[36] A. Ziletti, S. M. Huang, D. F. Coker, and H. Lin, Phys. Rev. B 92, 085423 (2015).

[37] M. S. Dresselhaus, G. Dresselhaus, and A. Jorio, Group Theory: Application to the Physics of Condensed Matter (SpringerVerlag, Berlin, 2008).

[38] J. Fabian and S. Das Sarma, Phys. Rev. Lett. 83, 1211 (1999).

[39] B. Zimmermann, P. Mavropoulos, S. Heers, N. H. Long, S. Blügel, and Y. Mokrousov, Phys. Rev. Lett. 109, 236603 (2012).

[40] N. H. Long, P. Mavropoulos, S. Heers, B. Zimmermann, Y. Mokrousov, and S. Blügel, Phys. Rev. B 88, 144408 (2013).

[41] B. Zimmermann, P. Mavropoulos, N. H. Long, C.-R. Gerhorst, S. Blügel, and Y. Mokrousov, Phys. Rev. B 93, 144403 (2016). 